\title{
Modeling Hydrogen Networks for Future Energy Systems: A Comparison of Linear and Nonlinear Approaches
}

Markus Reuß ${ }^{1}$, Lara Welder, ${ }^{1,6}$, Johannes Thürauf ${ }^{2,3}$, Jochen Linßen ${ }^{1}$, Thomas Grube ${ }^{1}$, Lars Schewe $^{4}$, Martin Schmidt ${ }^{5}$, Detlef Stolten ${ }^{1,6}$, Martin Robinius ${ }^{1}$

1) Forschungszentrum Jülich, IEK-3: Institute of Electrochemical Process Engineering, 52425 Jülich, Germany

2) Energie Campus Nürnberg, Fürther Str. 250, 90429 Nürnberg, Germany

3) Discrete Optimization, Friedrich-Alexander-Universität Erlangen-Nürnberg (FAU), Cauerstr. 11, 91058 Erlangen, Germany

4) University of Edinburgh, School of Mathematics, James Clerk Maxwell Building, Peter Guthrie Tait Road, Edinburgh, EH9 3FD, UK

5) Trier University, Department of Mathematics, Universitätsring 15, 54296 Trier, Germany

6) RWTH Aachen University, Chair for Fuel Cells, Faculty of Mechanical Engineering, Kackertstr. 9, D-52072 Aachen

\begin{abstract}
Common energy system models that integrate hydrogen transport in pipelines typically simplify fluid flow models and reduce the network size in order to achieve solutions quickly. This contribution analyzes two different types of pipeline network topologies (namely, star and tree networks) and two different fluid flow models (linear and nonlinear) for a given hydrogen capacity scenario of electrical reconversion in Germany to analyze the impact of these simplifications. For each network topology, robust demand and supply scenarios are generated. The results show that a simplified topology, as well as the consideration of detailed fluid flow, could heavily influence the total pipeline investment costs. For the given capacity scenario, an overall cost reduction of the pipeline costs of $37 \%$ is observed for the star network with linear cost compared to the tree network with nonlinear fluid flow. The impact of these improvements regarding the total electricity reconversion costs has led to a cost reduction of $1.4 \%$, which is fairly small. Therefore, the integration of nonlinearities into energy system optimization models is not recommended due to their high computational burden. However, the applied method for generating robust demand and supply scenarios improved the credibility and robustness of the network topology, while the simplified fluid flow consideration can lead to infeasibilities. Thus, we suggest the utilization of the nonlinear model for postprocessing to prove the feasibility of the results and strengthen their credibility, while retaining the computational performance of linear modeling.
\end{abstract}

\section{Keywords}

Hydrogen reconversion; Hydrogen infrastructure; Spatial resolution; Pipeline design optimization; Pressure drop; Robust optimization 


\section{Introduction}

The mitigation of greenhouse gas emissions to tackle climate change is one of the main challenges facing upcoming generations [1]. It is broadly accepted that high shares of renewable energies such as photovoltaic or wind power systems are necessary to replace fossil energy carriers [2]. However, the weather dependencies of renewables necessitate the implementation of large-scale storage systems. Hydrogen represents a chemical storage technology with high energy density and long-term stability. Thus, the production of hydrogen enables the transfer of renewable energy into other sectors like transport [3] or heat [4], which may otherwise struggle to reach their greenhouse gas reduction targets.

Several studies deal with the integration of hydrogen into the future energy system. Yang and Ogden [5], for instance, investigate hydrogen transport and determine that transport through pipelines offers the lowest transmission costs for demand-intensive scenarios. Baufumé et al. [6], in turn, design a nationwide hydrogen pipeline grid for supplying $75 \%$ of the German transport sector and demonstrate the economic feasibility of hydrogen pipelines. Reuß et al. [7], meanwhile, elaborate on different infrastructure technologies and show that the transmission of hydrogen via pipelines and its storage in underground formations like salt caverns are key technologies for future infrastructure development.

While these studies focus on hydrogen itself, the impact of integrating hydrogen in an energy system requires the presence of competitive technologies as well. Hence, the scientific community has developed various optimization models to evaluate the impact of hydrogen on the system as a whole. Welder et al. [8], for example, investigate a German hydrogen infrastructure with a spatio-temporal approach that focuses on the role of caverns for the infrastructure. In addition, the authors analyze an electrical reconversion scenario for the supply of North Rhine Westphalia and illustrate the important existence of hydrogen even with competing electricity transport [9]. Samsatli et al. [10,11] investigate the UK energy system's capacity to supply hydrogen to the domestic transport or heat sectors. Moreno-Benito et al. [12], in turn, consider different hydrogen production options for evaluating cost-optimal hydrogen infrastructure development in the UK through 2100, neglecting a temporal resolution. Almansoori and Betancourt-Torcat [13], as well as Ochoa Bique and Zondervan [14], compute a nationwide hydrogen supply chain for Germany, in which they do not take hydrogen pipelines and temporal resolution into account. Weber and Papageorgiou [15], meanwhile, minimize network costs with consideration of hydraulics in hydrogen pipelines. However, the authors do not consider a temporal resolution to address the fluctuations of demand and supply.

The more sectors an energy system optimization considers, the lower the degree of detail that is typically allowed for the representation of infrastructure due to limitations of model size and computational tractability. Welder et al. [8, 9] therefore consider pipelines for hydrogen transport but neglect the effects of pressure drops by applying a fixed gas velocity and a linearization of pipeline costs for varying hydrogen flows. Samsatli et al. [10] consider just one pipeline diameter and define the maximum flowrate during preprocessing without taking different diameters into account. Similarly, Moreno-Benito et al. [12] allow the selection of six discrete diameters with a predefined maximum flowrate but without taking the pressure drops into account. In addition, Welder et al. [8, 9], Samsatli et al. [10], and Moreno-Benito and Agnolucci [12] create regions for the spatial resolution and utilize distance matrices for the estimation of transport distances from region to region.

Groissböck [16] reviewed 33 open source energy system optimization models and showed that not a single model considers physical constraints like pressure as part of the optimization. Thus far, the consideration of pressure drops in system design was only implemented for stand-alone pipeline network models. This is mainly due to computational performance losses resulting from the nonlinear pressure drop behavior of pipeline sizing as well as the increasing problem size with higher numbers of nodes. Robinius et al. [17] thus present a novel approach to integrate the nonlinear pressure drop into a mixed-integer nonlinear pipeline design optimization model for tree-structured transport networks. To integrate the physical behavior 
of hydrogen, the authors further develop an approach used for operational optimization of the today's natural gas grid as used in Schmidt et al. [18, 19], see also Aßmann et al. [20]. To address fluctuating demand and production uncertainties, Robinius et al. [17] develop an algorithm for modeling "robust" demand scenarios. Optimizing with respect to these scenarios guarantees the feasibility of all balanced demands and supplies within previously given flow rates of sources, sinks, and storage options. Aside from these flow rates, the algorithm requires a predefined pipeline topology and the allocation of hydrogen sources, sinks, and storage options. The method is linked to the field of robust optimization, which aims at protecting an optimization problem from deterministic data uncertainties; see Gorissen et al. [21] for an introduction to robust optimization. In summary, the method is not suitable for optimizing the entire energy system. However, the robust demand and supply scenarios obtained allow for consideration of pressure drops in the entire network.

Samsatli et al. [10] and Moreno-Benito et al. [12] consider pressure drops during preprocessing to elaborate the maximum flowrate of each pipeline section. In theory, they require an additional compressor at each node to recompress the hydrogen to the maximum pressure level. With the nonlinear model of Robinius et al. [17], the pressure level in each pipe section is considered and made part of the optimization. As a result, the pipeline network is designed for avoiding compressor stations for recompression in the entire network.

Meanwhile, energy system optimization models with spatial resolution usually feature regionalization with a simplification of transport options for "region-to-region" energy transport $[8,10,12,13,15]$. This assumption highly simplifies the pipeline network, especially for low numbers of regions. In contrast, pipeline design models that have a high spatial resolution like those of Baufumé et al. [6] or André et al. [22] compute the pipeline design without taking the costs of production and storage into account. The error arising from simplifying the pipeline network design and, respectively, the topology, has not yet been investigated.

Energy system models often simplify pipeline topologies and fluid flow models. The focus of this work is to evaluate the impact of nonlinear pressure drop considerations, as well as the impact of topology design on hydrogen pipeline system feasibility and costs. To this end, we apply the model from Robinius et al. [17], which is briefly described in Subsection 2.1, to the pipeline topology and capacity scenario from Welder et al. [9] for two different network topologies, which are given in Subsection 2.2. In Section 3, the results regarding pipeline sizing, total system costs, differences between topologies, and flow modeling approaches are analyzed and discussed. Finally, the feasibility of the linear model is checked by a nonlinear model.

\section{Methods and Data}

This section introduces the methods used, as well as the data. In Figure 1, the two workflows for determining the pipeline topologies (Workflow I) and the resulting pipeline designs (Workflow II) for two different design methods are shown. Workflow I starts with a candidate pipeline grid that represents existing pipeline routes used for natural gas connected to the source, sink, and storage nodes of the demand and supply scenario. The geo-referenced pipeline data is taken from Welder et al. [9]. The candidate pipeline grid is then reduced to represent potential routes that could be selected during the optimization. Dijkstra's algorithm [23] is then used for creating shortest-path connections between source, sink, and storage nodes. The remaining grid represents the "tree network". To create the "star network", the real course of the pipes is neglected and, instead, centroid-to-centroid connections with one distance value for each connection are employed.

In Workflow II, the "capacity scenario" is first evaluated by performing an energy system optimization based on techno-economic input data and geo-referenced residual electricity loads. The capacity scenario consists of hydrogen injection and withdrawal time series and is taken from Welder et al. [9]. These time series are necessary for generating a robust scenario set according to the scenario generation algorithm of Robinius et al. [17]. As a result, demand 
and supply scenarios are generated for each pipeline topology, namely for the star network and tree network. Finally, two different approaches for modeling physical flow properties are applied. The linear model (LP) fixes the gas velocity and neglects the pressure as the driving force of fluid flow. The mixed-integer nonlinear model (MINLP), in turn, minimizes the total pipeline costs with respect to pressure drop in the pipes by using the discrete arc sizing optimization from Robinius et al. [17].

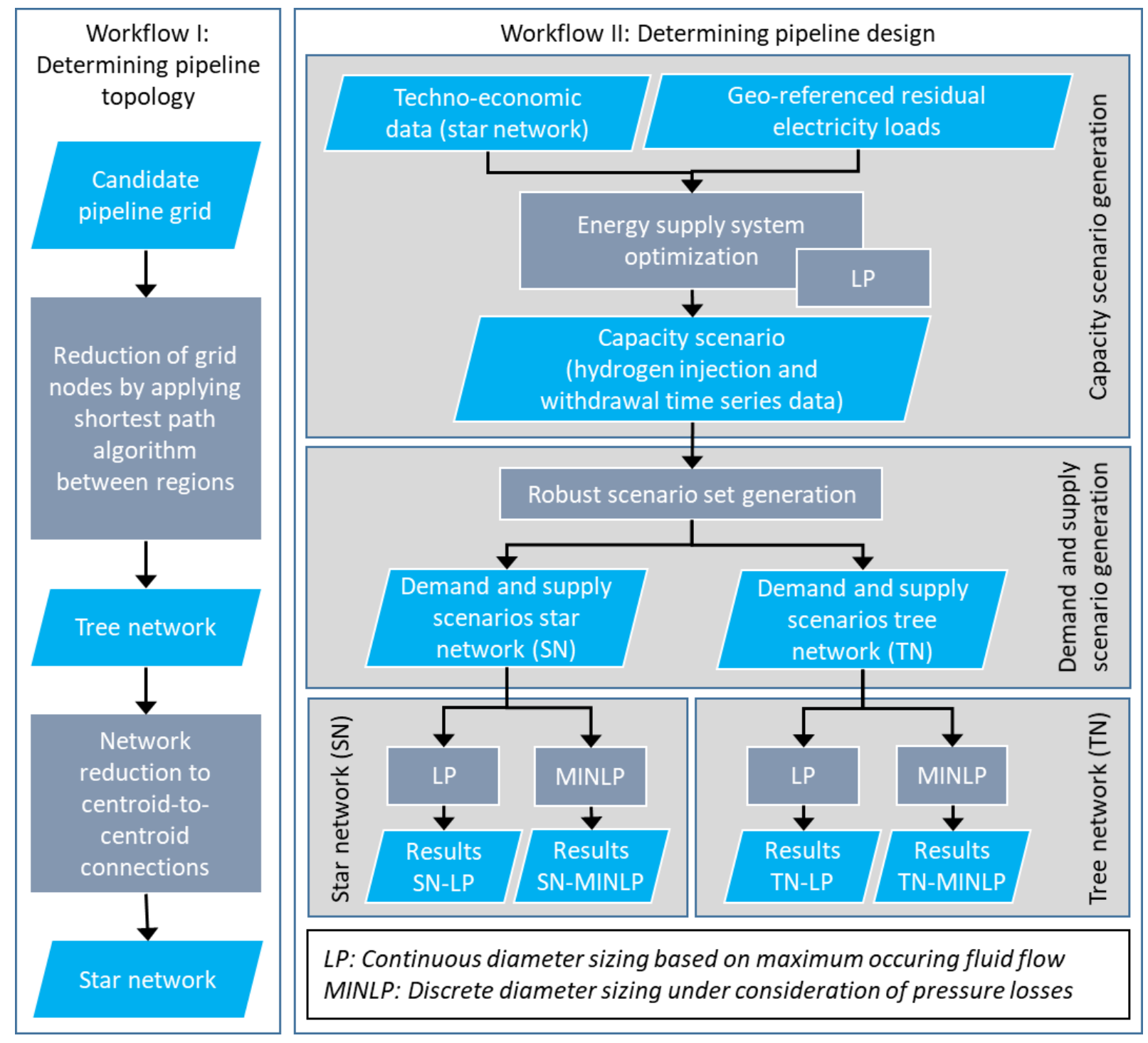

Figure 1. Explanation of the scenario and the workflows used in this study.

\subsection{Pipeline Design Modeling}

The modeling of the pipeline design uses either a linear or nonlinear fluid flow representation and aims to minimize the total pipeline costs.

The nonlinear model used in this study for minimizing the pipeline costs is developed and explained in detail in Robinius et al. [17]. The pressure drop is considered by using the Weymouth equation [18, 19, 24-26] for the approximated relationship between mass flow, diameter, and gas pressures. As the pipeline investment costs are mainly dependent on the pipeline diameters, the overall system costs are optimized by selecting the diameters.

Aside from the medium (hydrogen), the main inputs for the model are the lower and upper pressure bounds and the discrete pipeline diameters. All pipelines are considered to operate between 70 and 100 bar. Pipeline diameters beyond DN1400 are not considered, as the 
application of looped pipes is more reasonable for such diameters. As the model can become infeasible without options for higher hydrogen flows, the method of Lenz and Schwarz [27] is used to calculate the equivalent pipeline diameter $D$ for two looped pipes $D_{1}$ and $D_{2}$ :

$$
D=\left(D_{1}^{\frac{5}{2}}+D_{2}^{\frac{5}{2}}\right)^{\frac{2}{5}} \text {. }
$$

The investment costs for the discrete diameters of the pipeline are considered according to an empirical cost analysis for natural gas pipelines designed for a pressure level of 100 bar from Mischner et al. [28]. The specific pipeline investment costs are dependent on the pipeline's diameter $\mathrm{D}$ in $\mathrm{mm}$. As Mischner et al. [28] give pipeline costs for natural gas pipelines, their utilization with hydrogen is conservatively estimated to have $5 \%$ higher costs, similar to Welder et al. [9],

$$
\text { Invest }=1.05 \cdot 278.24 \cdot e^{1.6 \cdot D},
$$

with pipeline diameter $D$ in $\mathrm{mm}$ and specific investment costs in $E U R / m$.

The considered pipeline diameters and resulting specific investment costs are given in Table 1.

Table 1. Considered pipeline diameters and respective investment costs.

\begin{tabular}{lcc|ccc} 
Name & $\begin{array}{c}\text { Diameter } \\
(\mathbf{m m})\end{array}$ & $\begin{array}{c}\text { Investment costs } \\
(\text { EUR/m) }\end{array}$ & Name & $\begin{array}{c}\text { Diameter } \\
(\mathbf{m m})\end{array}$ & $\begin{array}{c}\text { Investment costs } \\
\text { (EUR/m) }\end{array}$ \\
DN100 & 106 & 346 & DN750 & 769 & 999 \\
DN125 & 131 & 360 & DN800 & 814 & 1.075 \\
DN150 & 159 & 377 & DN850 & 864 & 1.164 \\
DN200 & 207 & 407 & DN900 & 915 & 1.263 \\
DN250 & 259 & 442 & DN950 & 960 & 1.357 \\
DN300 & 306 & 477 & DN1000 & 1011 & 1.473 \\
DN325 & 336 & 500 & DN1050 & 1058 & 1.588 \\
DN350 & 384 & 540 & DN1100 & 1104 & 1.709 \\
DN400 & 432 & 583 & DN1150 & 1155 & 1.854 \\
DN450 & 480 & 629 & DN1200 & 1249 & 2.155 \\
DN500 & 527 & 679 & DN1300 & 1342 & 2.501 \\
DN550 & 578 & 737 & DN1400 & 1444 & 2.944 \\
DN600 & 625 & 794 & 2xDN1200 & 1648 & 4.311 \\
DN650 & 671 & 855 & 2xDN1300 & 1771 & 5.002 \\
DN700 & 722 & 927 & 2xDN1400 & 1905 & 5.889
\end{tabular}

According to Samsatli et al. [29], the gas velocity inside pipelines is not only limited by pressure drop, but also by mechanical interferences. Excessively high gas velocities could lead to erosional behavior or high noise emissions. Therefore, the gas velocity must be limited. Although the pressure drop consideration itself leads to a limited possible gas velocity, an upper bound for the gas velocity is not implemented in the model of Robinius et al. [17]. Therefore, we added a constraint to the model that the velocity $v$ in each pipeline must be less than or equal to compared to the predefined maximum gas velocity $v_{\text {max }}$.

For the linear case, the occurrence of a pressure drop inside the pipeline is neglected and a constant gas velocity is assumed, as in Welder et al. [9]. The corresponding pipeline costs are based on Equation (2). Welder et al. [9] linearize the pipeline investment after fixing the gas velocity inside the pipeline to $10 \mathrm{~m} / \mathrm{s}$ and assume a fixed gas density of $5.7 \mathrm{~kg} \mathrm{H}_{2} / \mathrm{m}^{3}$, similar to other studies $[6,8,12]$, to keep the overall optimization problem solvable through a mixed- 
integer linear programming solver. The pipeline capacity is linked to the hydrogen flow by using the lower heating value of hydrogen of $33.32 \mathrm{kWh} / \mathrm{kg}_{\mathrm{H} 2}$, where $1 \mathrm{GW}_{\mathrm{H} 2}$ consequently corresponds to $8.34 \mathrm{~kg} \mathrm{H}_{2} / \mathrm{s}$,

$$
\text { Invest }_{\text {linear }}=180 \cdot P+408 \text {, }
$$

with a pipeline capacity $P$ in $\mathrm{GW}_{\mathrm{H} 2}$ and specific pipeline investment Invest $_{\text {linear }}$ in $(\mathrm{EUR} / \mathrm{m})$.

In Figure 2, the linearized cost function from Equation (3) is compared to the basic costs function from Equation (2). While the linearization for low hydrogen flows slightly overestimates the investment costs, the general behavior is represented quite well. However, the velocity is fixed for that purpose, which is not the case for models considering a pressure drop.

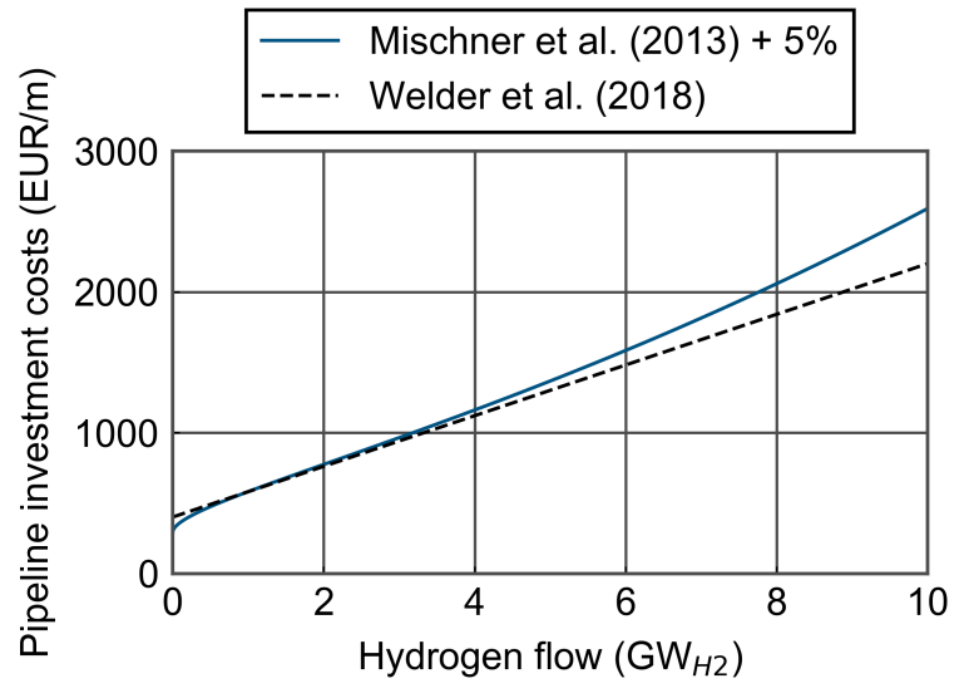

Figure 2. Adjusted pipeline investment costs from Mischner et al. [28], Equation (2), for gas velocity (10 m/s) and density $\left(5.7 \mathrm{kgH}_{2} / \mathrm{m}^{3}\right)$ compared to the linearized cost function from Welder et al. [9], Equation (3), depending on the maximum flow.

To calculate the pipeline-specific expenditures as part of the levelized costs of electricity, a depreciation period for the pipeline of 40 years as well as a yearly operation and maintenance of 5 EUR per meter and year is assumed. The weighted average cost of capital is set to $8 \%$.

\subsection{Capacity Scenario and Pipeline Topologies}

The capacity scenario for this study is based on an energy system design with respect to hydrogen reconversion pathways according to Welder et al. [9]. The aim of the authors' system design is to cover the positive residual load at every point in time and in every region of the German federal state of North Rhine-Westphalia (NRW) in the year 2050 by means of surplus electricity from northern Germany. The underlying residual load data from Robinius et al. [30] is used, who design a German energy system for the year 2050 with spatially-resolved production and demand data to supply most of the German electricity sector with renewable energies. The major share of renewable electricity is produced from wind onshore and offshore and is located in northern Germany. Therefore, the negative residual load is located in northern districts as well. More details about the underlying scenario like the spatially-resolved installed capacities of renewable energy technologies with corresponding temporal electricity generation profiles are given in Robinius et al. [30] and Welder et al. [9].

Welder et al. [9] analyze hydrogen reconversion pathways with spatially- and temporallyresolved load data in which energy transfer using the necessary infrastructure (hydrogen pipelines and underground high-voltage direct-current transmission cables) is integrated in the model. The system design is shown in Figure 3. 


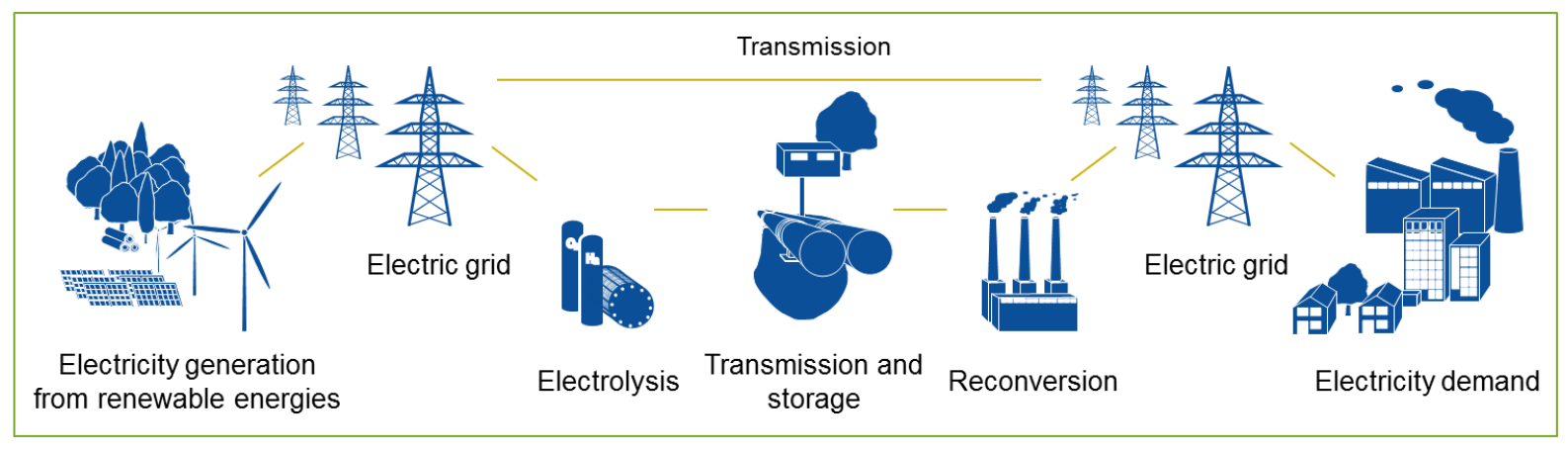

Figure 3. Schematic of hydrogen reconversion pathway for supplying North Rhine Westphalia (NRW) with electricity, reproduction from Welder et al. [9], with permission from Elsevier.

As potential reconversion options, Welder et al. [9] evaluated reconversion by combined cycle gas turbines (CCGT), solid oxide fuel cells, gas motors, polymer electrolyte membrane fuel cells, and gas turbines. According to their results, electrification by means of CCGT is the most promising pathway with respect to economic boundaries. For our study, we focus on the pathway utilizing CCGT from Welder et al. [9]. In Figure 4, the resulting capacities for the CCGT pathway are visualized.

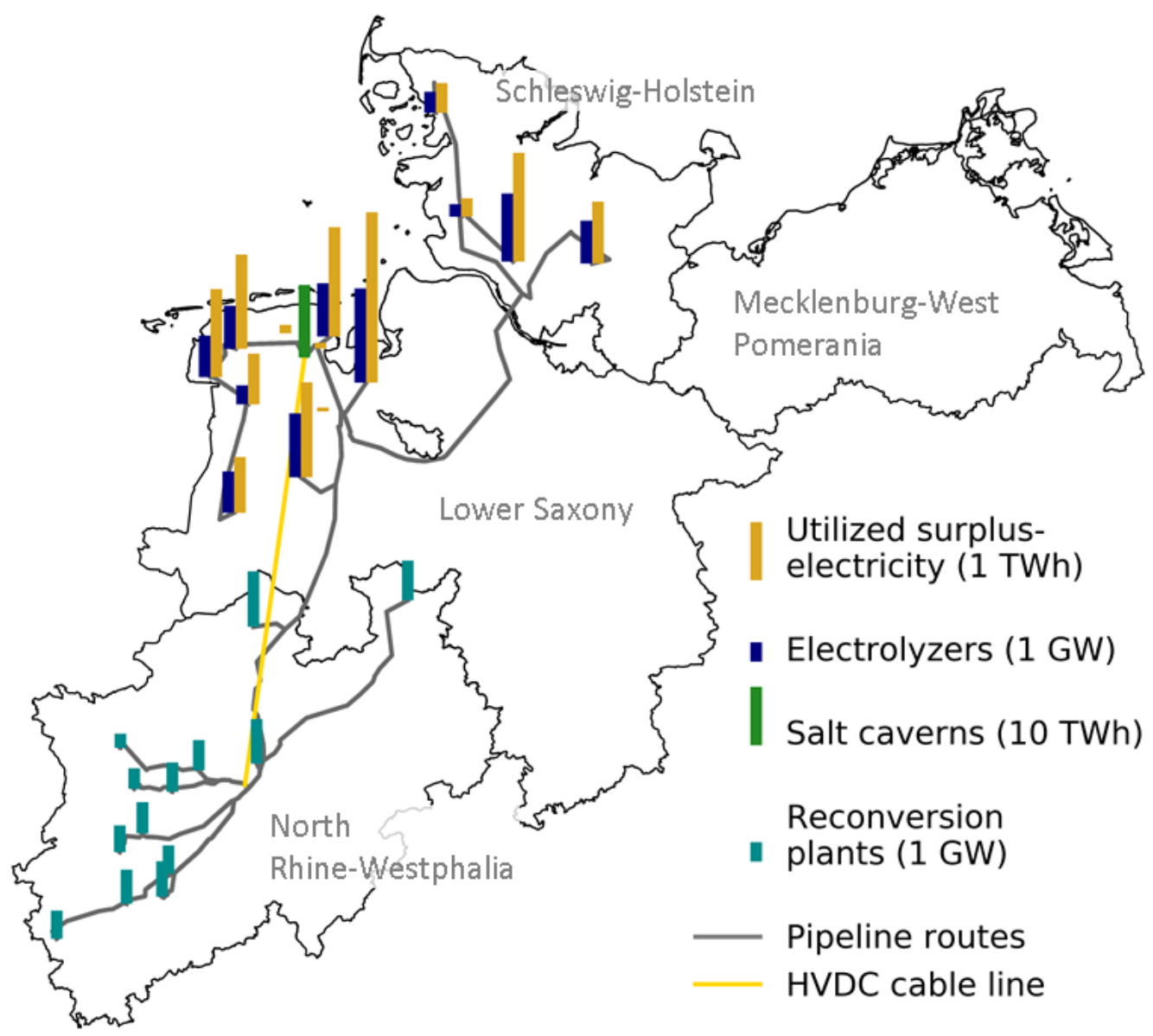

Figure 4. Installed capacities of the CCGT reconversion power plants, storage capacity of the salt caverns, surplus electricity used, and the pipeline routes, as well as HVDC routes in the cost-optimized reconversion pathway, reproduction from Welder et al. [9], with permission from Elsevier.

The pathways from Welder et al. [9] are modeled and optimized with the optimization framework FINE (Framework for Integrated Energy System Assessment) [31]. Within FINE, the hydrogen pipeline costs are integrated into the energy system optimization framework by 
determining the distances from all electrolysis locations to the salt cavern storage, the salt cavern storage to the centroid of NRW, and from the centroid of NRW to the CCGT locations via Dijkstra's shortest path algorithm [23]. The algorithm is utilized to route new pipelines next to existing infrastructure like the natural gas grid, motorways, and railway tracks. Based on these distances, the model is free to specify the pipeline diameters.

Even if the pipeline routes from Figure 4 look like a network merging multiple parallel pipes into single ones, the model itself does not take this into account and opts for a diameter for each connection. The shortest path distances are integrated to avoid the utilization of detour factors, but are not taking into account multiple parallel pipes. From the view of the model framework, the pipeline structure looks like that which is sketched in Figure 5, on the left side. While this star network has point-to-point connections between the key elements, the network, as shown in Figure 4, could be elaborated in much more detail by separating the pipeline routes into smaller sections and adding nodes where the pipes are forking, as is shown on the right-hand side of Figure 5, the tree network.
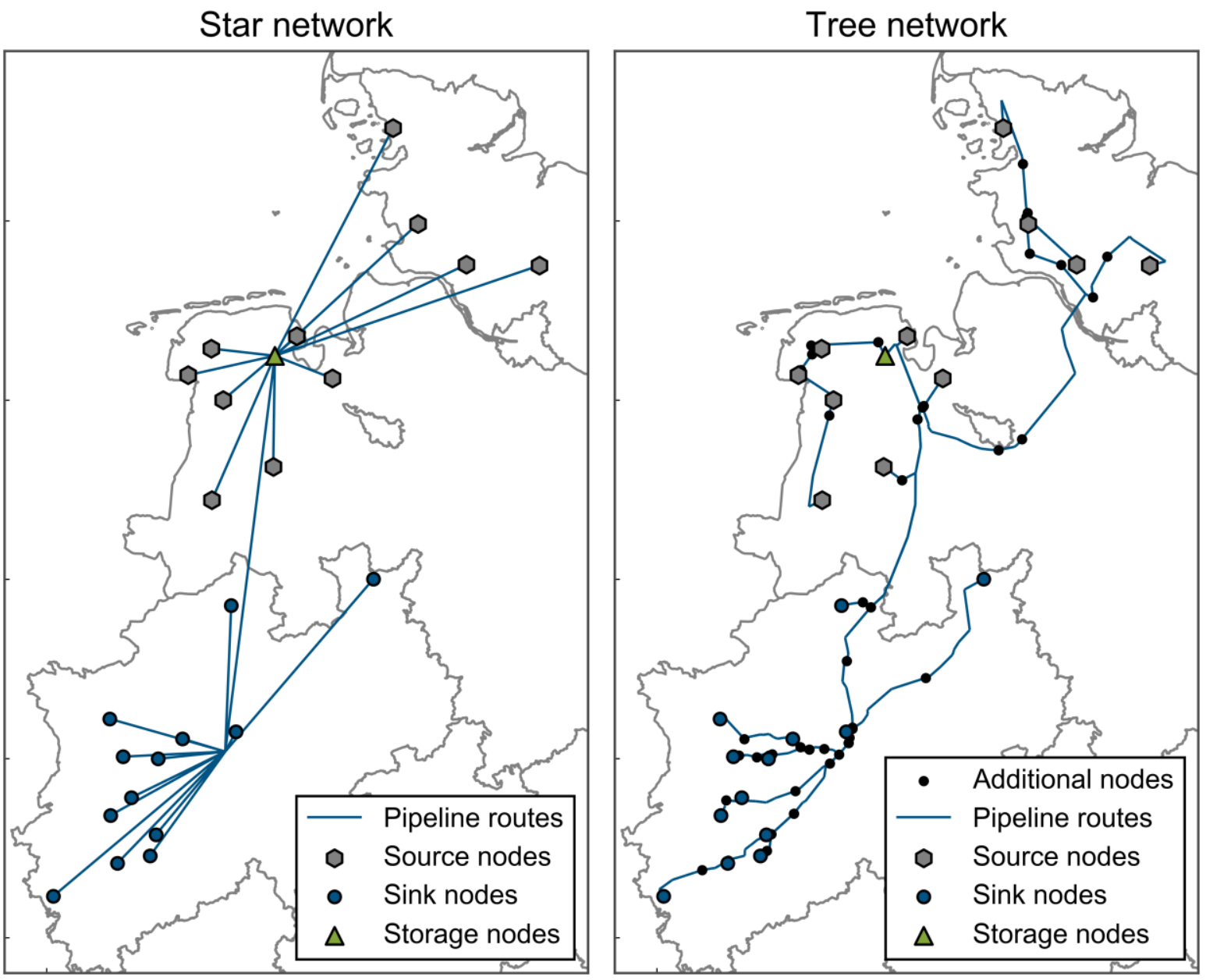

Figure 5. Model view on the pipeline network. Left: star network with connections from electrolysis to storage, from storage to the centroid of NRW, and from the centroid of NRW to power plant locations. Right: tree network with a fine pipeline grid.

\subsection{Demand and Supply Scenarios}

In the context of renewable energy technologies, demand and supply is affected by uncertainties such as weather conditions. Consequently, demand and supply fluctuations have to be considered. Robinius et al. [17] present an algorithm that tackles demand and supply uncertainties by constructing finitely many "robust" scenarios for a given tree-structured 
pipeline network. Here, "robust" means optimizing with respect to these scenarios, guaranteeing a feasible solution for all balanced demand and supply scenarios within given capacities of sources, sinks, and storage options. For the generation of these "robust" scenarios at most quadratically many (in terms of the number of nodes of the considered network), linear optimization problems must be solved, which can be done efficiently, i.e., in polynomial time. In this study, we compute the "robust" demand and supply scenarios with the help of the method presented in Robinius et al. [17] for the star network, as well as for the tree network. These scenarios form the basis of the following computational results.

\section{Results and Discussion}

In this section, the results for each pipeline topology as well as the linear and nonlinear fluid flow model are presented. Afterward, the results are compared and discussed.

\subsection{Analysis of the Star Network}

First, the spatially-resolved results for the star network are shown on the left-hand side of Figure 6. These results show that for each connection, from electrolysis to the storage, as well as the connections from the centroid of NRW to the locations of the CCGT, the selected diameters remain fairly small, with DN900 being the largest. The looped connection from the storage to the centroid of NRW has by far the largest pipeline diameter, as it is the main connection between north (production) and south (demand).

Within the scenario evaluation, a scenario set of 35 different scenarios are shown to be sufficient for a robust design of the network according to Section 2.3. On the right-hand side of Figure 6, the total investment of the pipeline network separated by diameter sizes is given. For the nonlinear pressure drop approach, a total investment of 3.77 billion EUR is computed. The linear approach, according to Welder et al. [9], results in pipeline costs of 3.93 billion EUR, which represents an overestimation of roughly $3.4 \%$. It is obvious that especially high diameters are overestimated by the linear model.

In Figure 7, the specific pipeline investment, the maximum occurring gas velocity, and the diameters of each pipeline section are drawn depending on their maximum hydrogen flow and compared for the nonlinear and linear models. The results for low hydrogen flows are similar for the linear and nonlinear model. Only the pipe with the highest hydrogen flow, which is, according to Figure 6, the pipeline between the centroid of NRW and the storage, has a significantly lower diameter in the nonlinear model compared to the linear approach. This is justified by the pressure drop consideration that allows for higher gas velocities compared to the linear approach. The view of gas velocities exposes the reason for the cost distribution in Figure 6: low hydrogen flow rates tend to have lower gas velocities than $10 \mathrm{~m} / \mathrm{s}$, while the velocity increases for high flow rates are up to $18 \mathrm{~m} / \mathrm{s}$. 


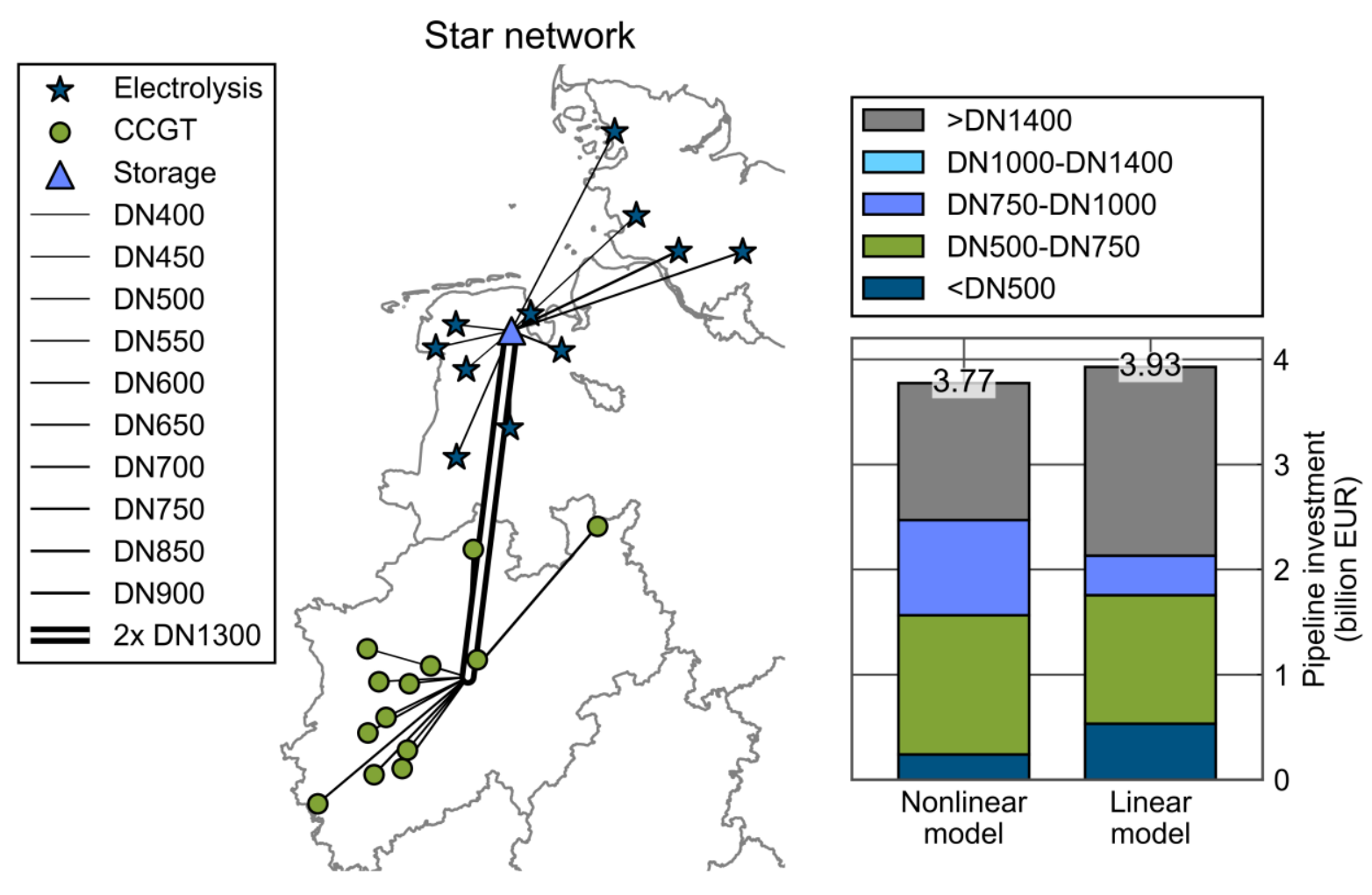

Figure 6. Spatially-resolved results of the star network (left) and pipeline investment distribution separated by pipeline diameters (right).

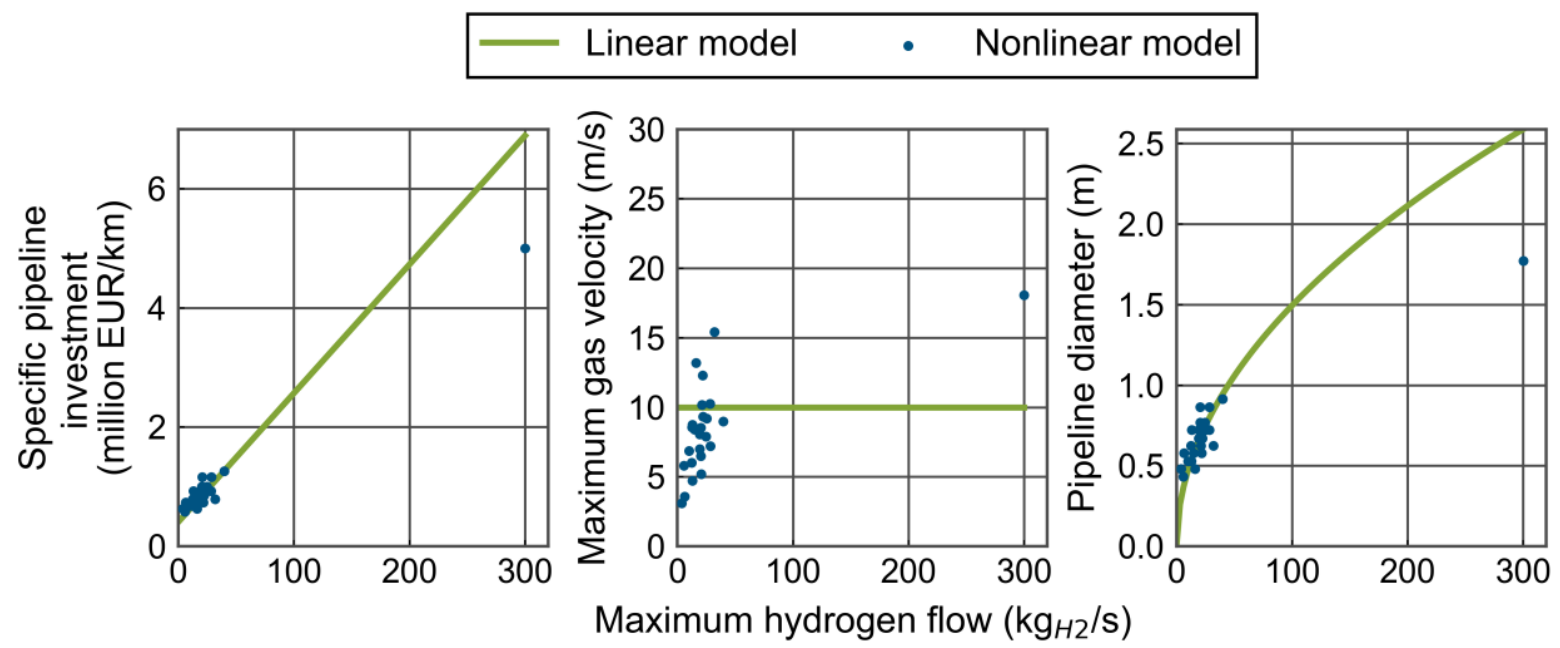

Figure 7. Specific costs, maximum gas velocities, and the diameters of each pipeline section depending on its maximum hydrogen flow in $\mathbf{3 5}$ scenarios for the star network results.

During the optimization, the pressure gradients for 35 scenarios are calculated to consider the pressure drop in each pipeline section. Figure 8 shows the pressure gradients of scenario 2 , which has the lowest pressure difference, and scenario 21 , which has the largest one. Scenario 2 only has a single connection between production and storage, which does not cause a notable hydrogen flow and therefore keeps the pressure drop low. In contrast, scenario 21 has very high demand and therefore requires a high outflow from the storage. This causes a high pressure drop on the connection from the storage to the centroid of NRW. 
Scenario 2

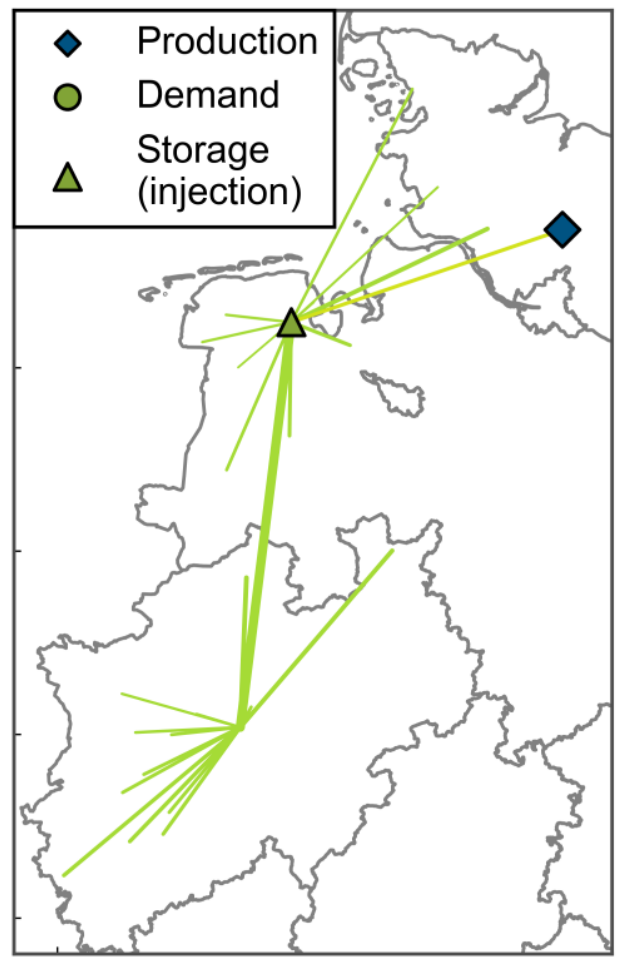

Scenario 21

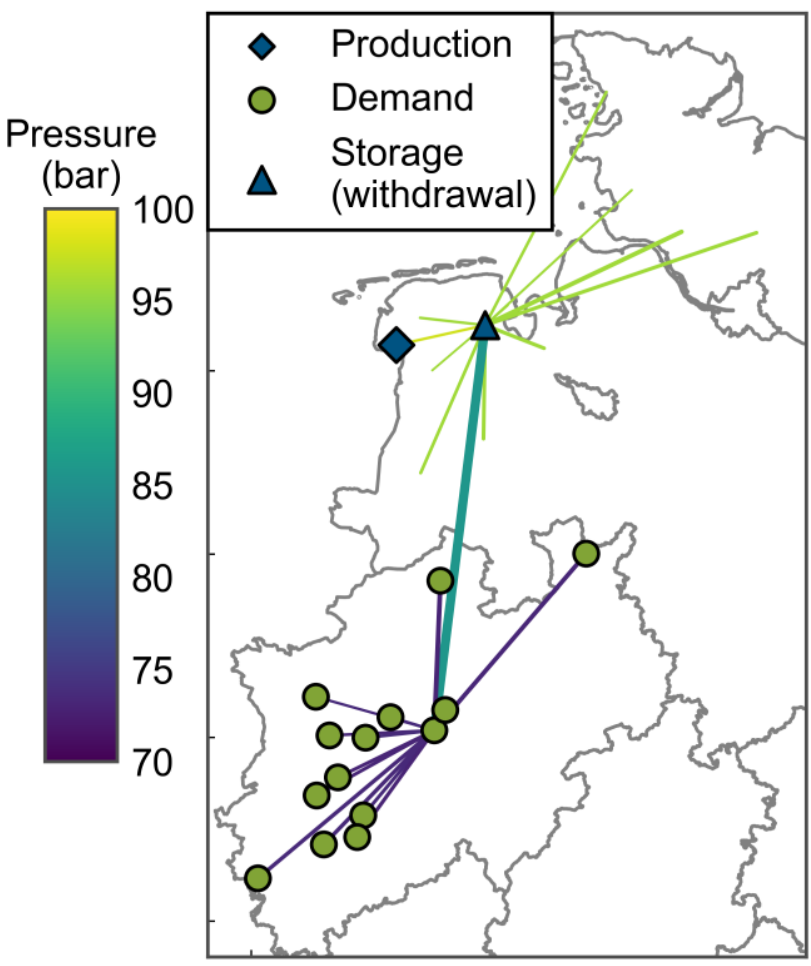

Figure 8. Exemplary pressure gradients of the star network for the low-demand scenario 2 and highdemand scenario 21.

\subsection{Analysis of the Tree Network}

The scenario generation resulted in 23 scenarios that are suitable for producing a robust solution for the tree network. The spatially-resolved results for the tree network are shown on the left-hand side of Figure 9. The results show that there remains a main backbone pipeline, as in Figure 6, from the storage in the north to the centroid of NRW. Nevertheless, single pipes are now merged together and the cost-optimal solution shows a larger variety of selected diameters. Even pipeline diameters between DN900 and DN1400 are now selected due to the merging of specific pipes to higher diameters. Analyzing the total investment costs on the righthand side of Figure 9, is apparent that the total costs of the pressure drop approach sums of up to 2.47 billion EUR. In contrast, the linear approach for the tree network results in 2.94 billion EUR. This represents an overestimation of costs by the linear model of $20 \%$.

Figure 10 gives insight into the reasons for the large benefit of the nonlinear pressure drop consideration. As already mentioned, there are now more pipelines with larger diameters. While the linear model selects for all pipes with the same maximum hydrogen flow the same diameter, the nonlinear model allows for different diameters. These tend to have lower specific costs compared to the linear model, as the maximum velocities are again increasing for increasing flow rates. While the maximum velocity occurring in Figure 7 is roughly $18 \mathrm{~m} / \mathrm{s}$, the maximum velocity for the tree network rises to almost $30 \mathrm{~m} / \mathrm{s}$, which is set as a limit. This is caused by having pipelines with smaller distances compared to the star network and is followed by more options for the solver to find cheaper solutions.

Figure 11 showcases the pressure gradients for the tree network of scenario 2, which has the lowest pressure difference, and scenario 3, which has the largest one. Scenario 2 has, in contrast to Figure 8, multiple locations of production that load the storage. Scenario 3 has, similarly to Figure 8 , a very high demand and therefore requires a high outflow from the storage. 


\section{Tree network}

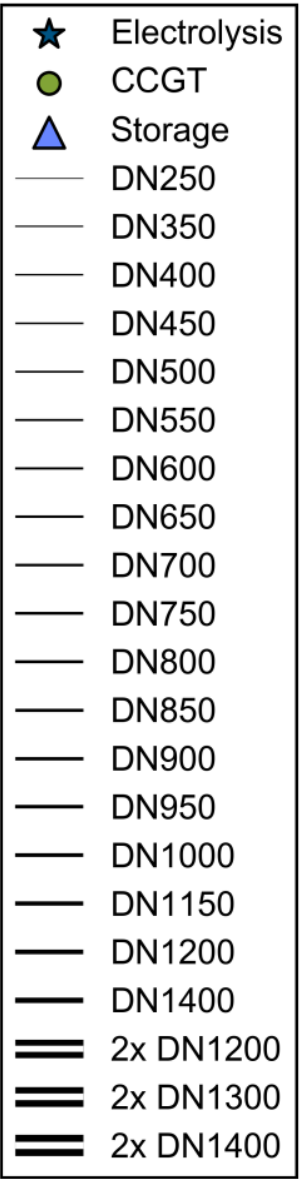

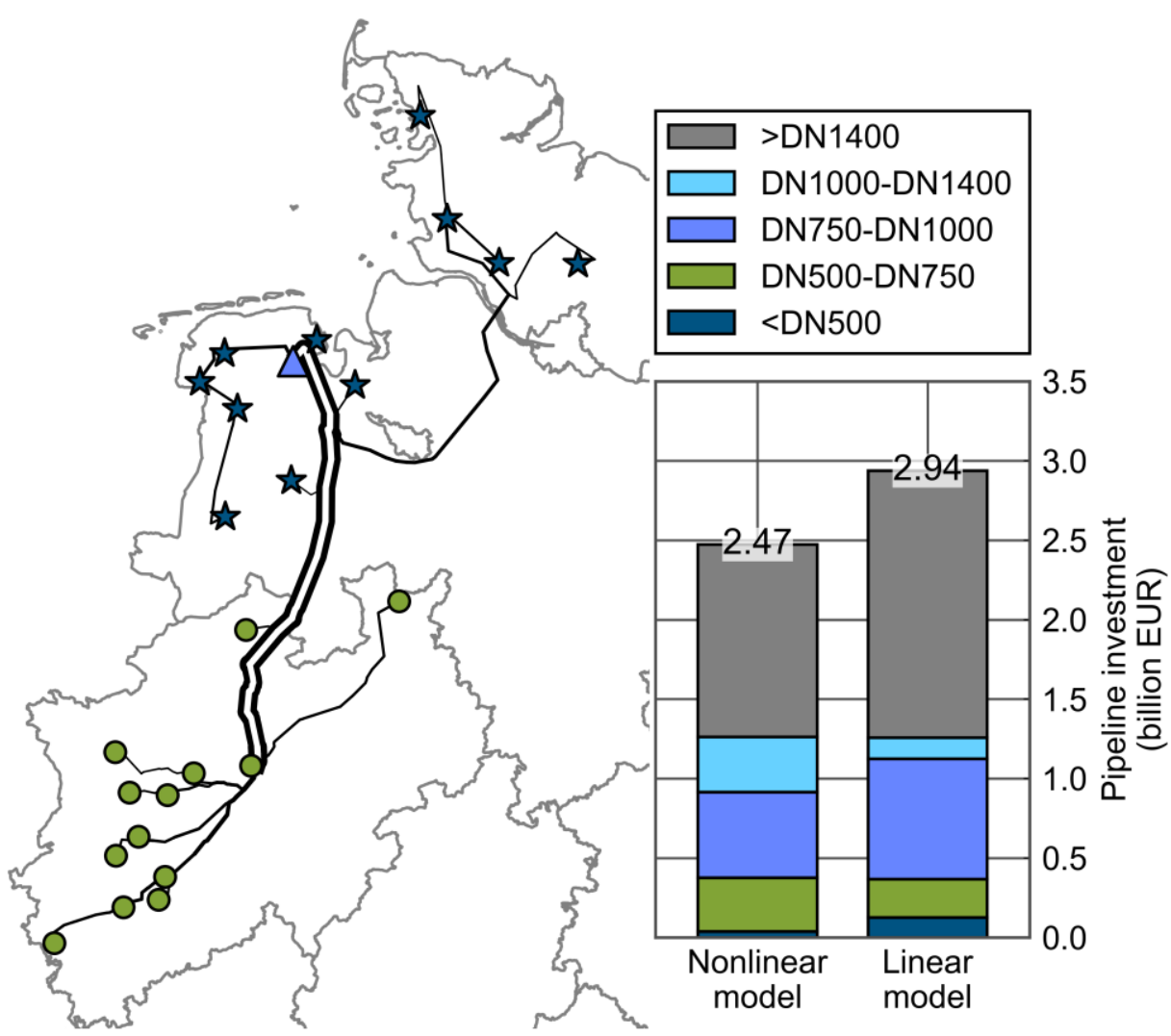

Figure 9. Spatially-resolved results of the tree network (left) and pipeline investment distribution separated by the pipeline diameters (right).

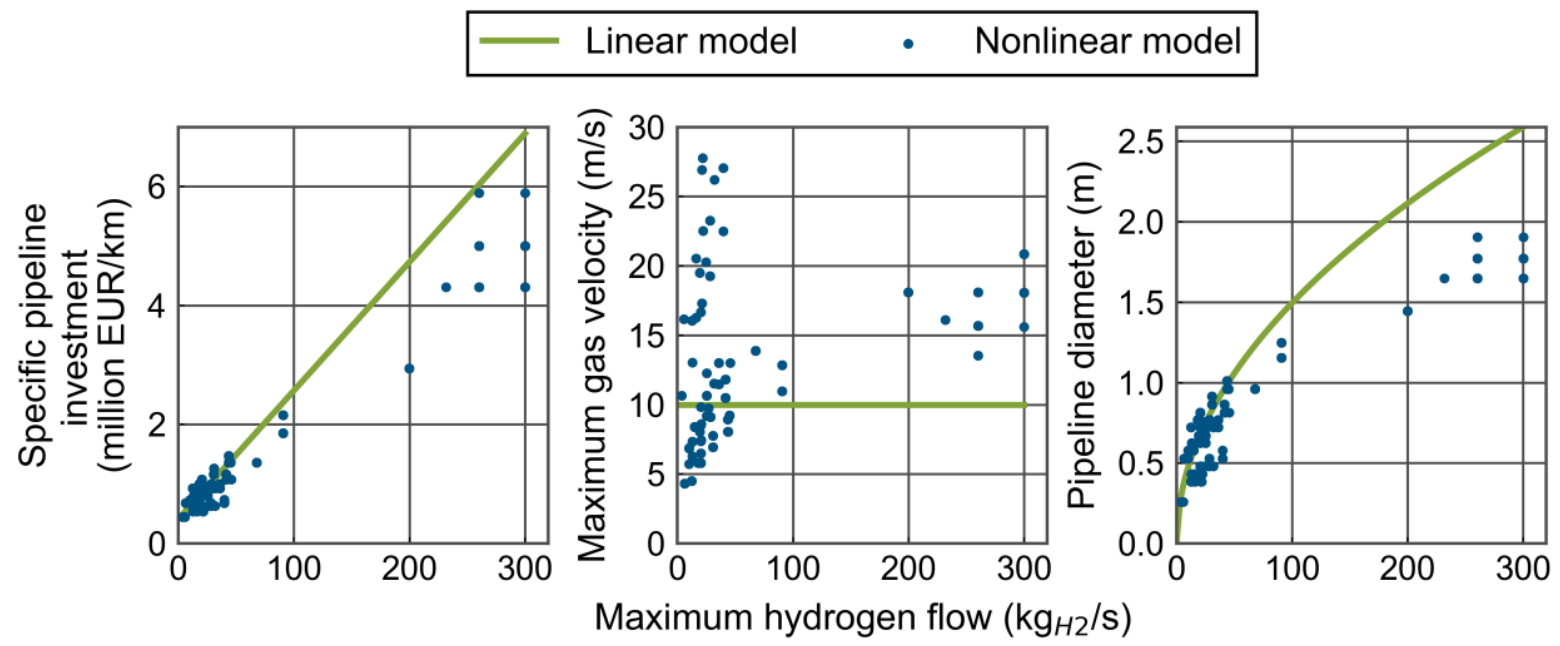

Figure 10. Specific costs, maximum gas velocities, and diameters of each pipeline section depending on its maximum hydrogen flow in 23 scenarios for the tree network results. 
Scenario 2

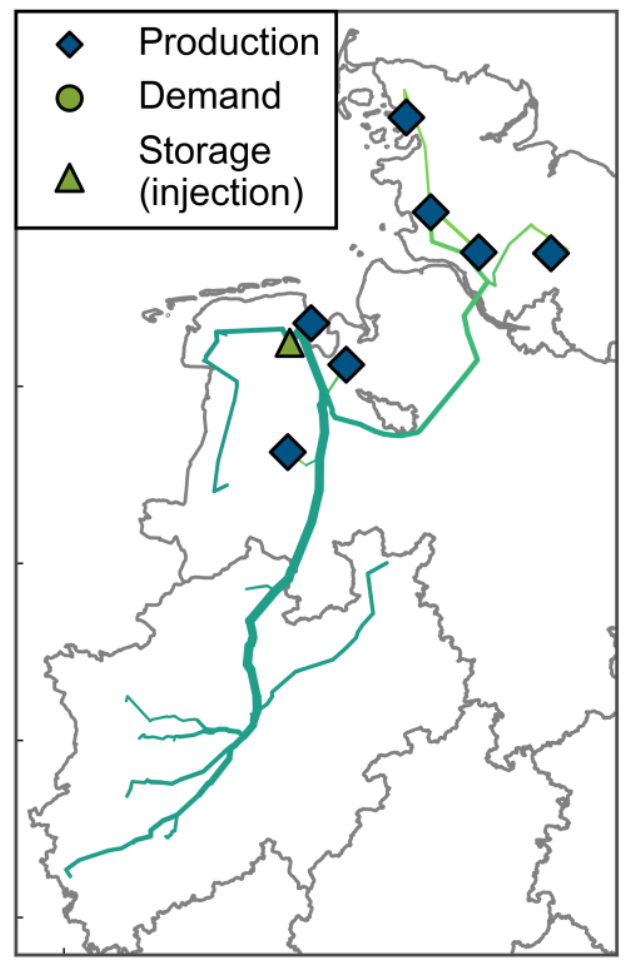

Scenario 3

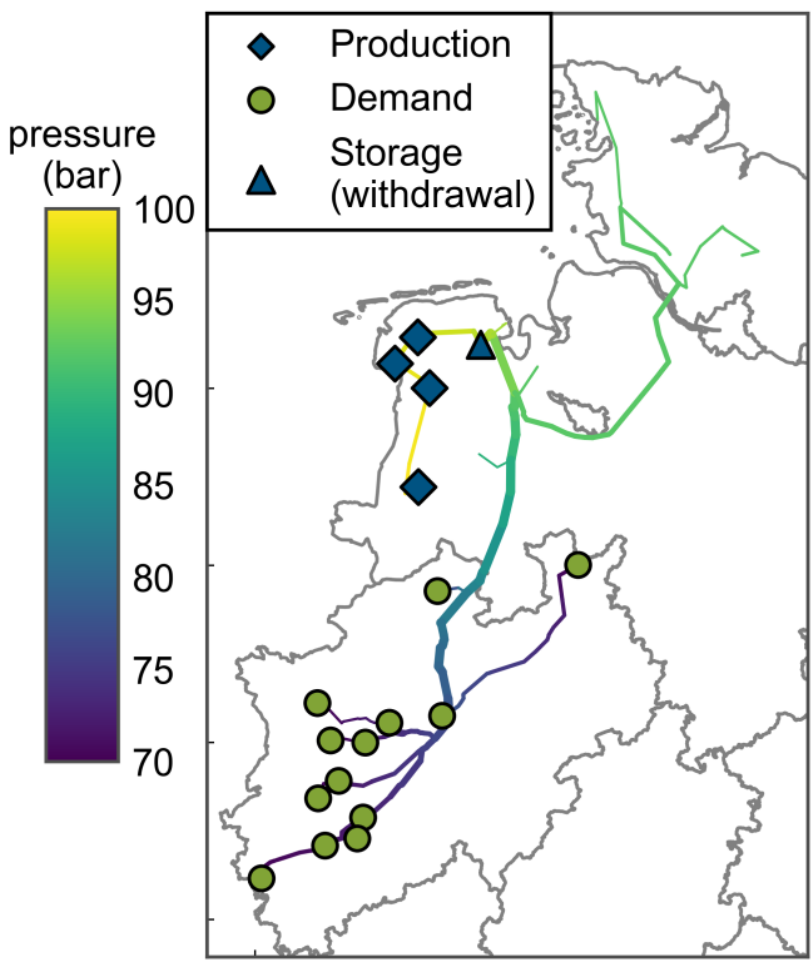

Figure 11. Exemplary pressure gradients of the tree network for scenario 2 (lowest pressure gradient) and scenario 3 (largest pressure gradient).

\subsection{Comparison and Discussion}

For a final comparison, Figure 12 shows the results for the total investment costs caused by both network topologies in comparison. It is obvious that the tree network assumption allows for a huge reduction in the pipeline investment requirement, even for the linear approach. This is caused by forking the network instead of using source-sink connections and is explained by Figure 2: two looped pipes of $2 \mathrm{GW}_{\mathrm{H} 2}$ would account to $766 \mathrm{EUR} / \mathrm{m}$ each (1524 EUR/m in total), while a $4 \mathrm{GW}_{\mathrm{H} 2}$ pipe would account for $1122 \mathrm{EUR} / \mathrm{m}$ and is, thus, $26 \%$ cheaper. The fixed cost share of 402 EUR, according to Welder et al. [9] and stated in Equation (3), especially impacts low pipeline diameters and affects every meter per pipeline built. As Figure 6 revealed, roughly $50 \%$ of the pipeline costs of the star network using the linear flow model are caused by pipelines with maximum occurring hydrogen flows below $5 \mathrm{GW}_{\mathrm{H} 2}$. In addition, bi-directional gas flows do not occur in the star network due to the topology. However, they occur within the tree network, which decreases the number of required pipes even more. These aspects lead to a large cost decrease for the linear approach from the star network to the tree network of roughly $25 \%$ of the total investment costs.

Figure 13 presents the length of the considered pipes obtained with the nonlinear model. Due to the parallel pipes consideration of the star network, the total pipeline length sums up to $3069 \mathrm{~km}$, whereas $91 \%$ of this total length belongs to small diameter pipelines. The merging of these pipes in the tree network reduces the total pipeline length to $1451 \mathrm{~km}$, which is only $47 \%$ of the star network's length and represents the main cost driver in Figure 12. 

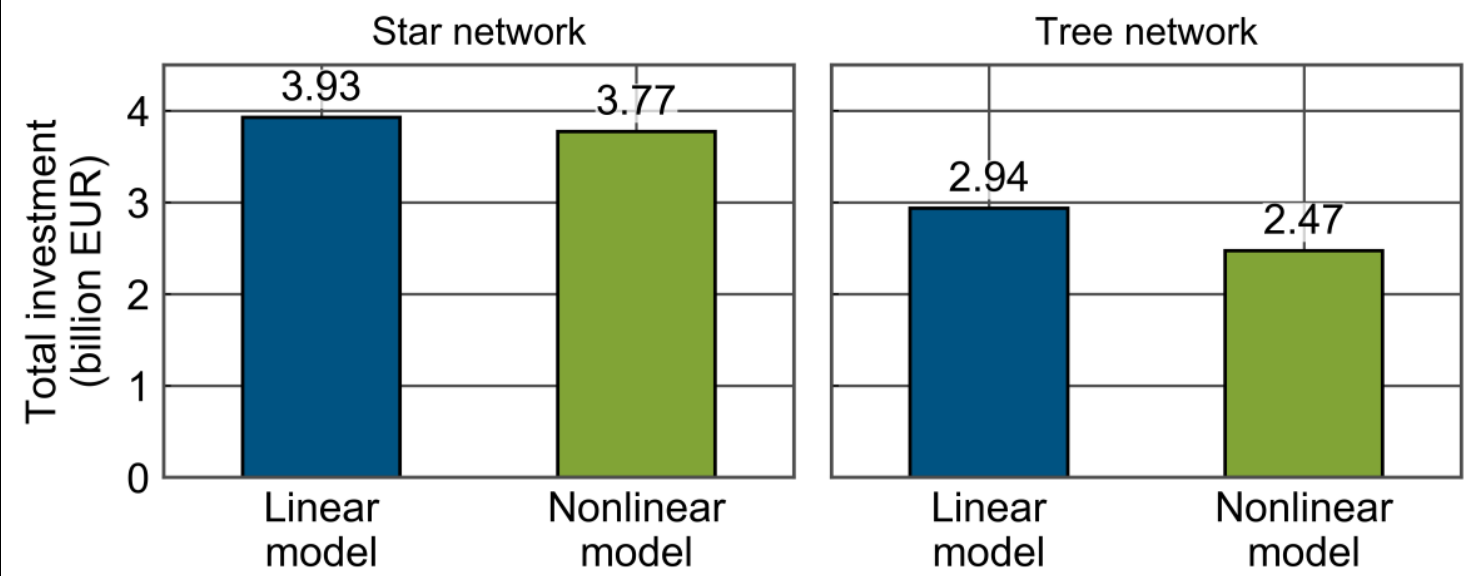

Figure 12. Comparison of pipeline investment costs for the two different networks and the two different flow modeling approaches.

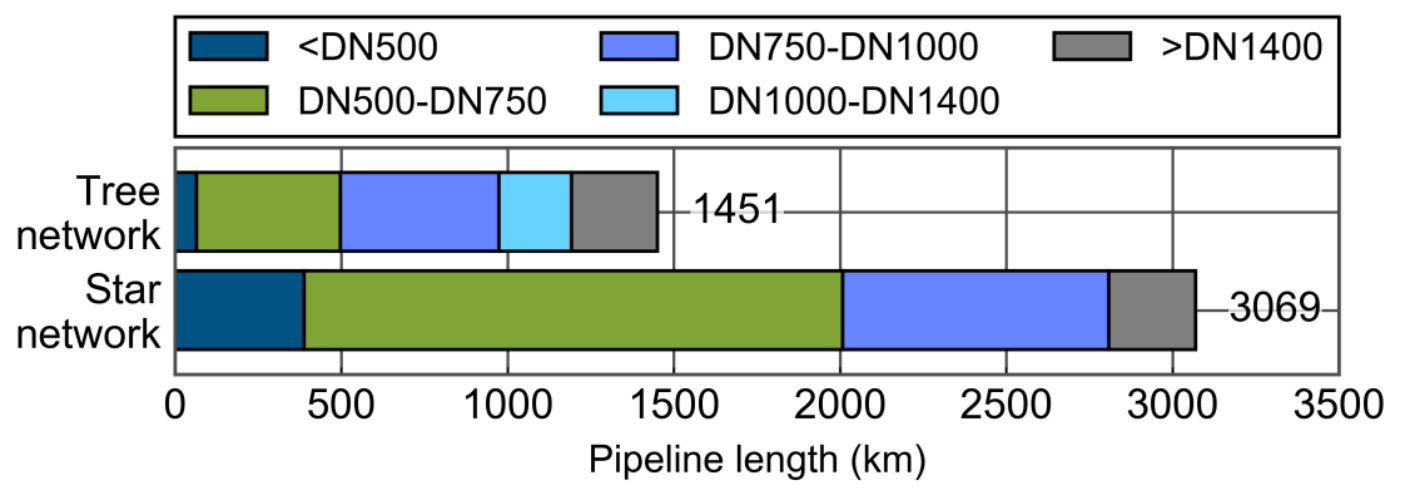

Figure 13. Comparison of the pipeline lengths of the two different networks considered.

As is shown in Figure 7 and Figure 10, the linear approach underestimate the costs for low flow rates compared to the pressure drop approach, while larger flow rates are overestimated. With regard to the star network, the total investment is balanced out and the relative difference between the linear and nonlinear model is below $5 \%$. In contrast, the tree network has pipelines with larger diameters instead of parallel ones. The average selected diameters of all pipes is higher compared to the star network. This overweighs the cost advantage of high diameters, as discussed in Figure 2 for the nonlinear approach and allows for a cost reduction for the tree network of an additional $16 \%$ compared to the linear model. Comparing the linear modeling applied to the star network with the nonlinear modeling in the tree network, a total cost reduction from 3.93 billion EUR to 2.47 billion EUR occurs, which amounts to $37 \%$. This impact reveals a large reduction in the total investment requirements, keeping in mind that the distances and pipes are based on the same topology.

Furthermore, a comparison of the specific hydrogen costs is shown in Figure 14. Based on the scenario from Welder et al. [9], a total electricity demand of 51.7 TWh is produced for electrical reconversion. As the specific pipeline costs are mainly driven by the capital expenditure (CAPEX), the cost differences from the linear model on the star network compared to the nonlinear model on the tree network are roughly $37 \%$. Comparing the specific pipeline costs with the overall electrical reconversion costs from Welder et al. [9] of 176 EUR per $\mathrm{MWh}_{\mathrm{el}}$, a fairly small impact of the pipeline costs for the entire energy system is revealed. The improvements made using the tree network, from 6.7 to $4.2 \mathrm{EUR} / \mathrm{MWh}$, account for $1.4 \%$ of the cost reduction for the entire electrical reconversion compared to the star network. 

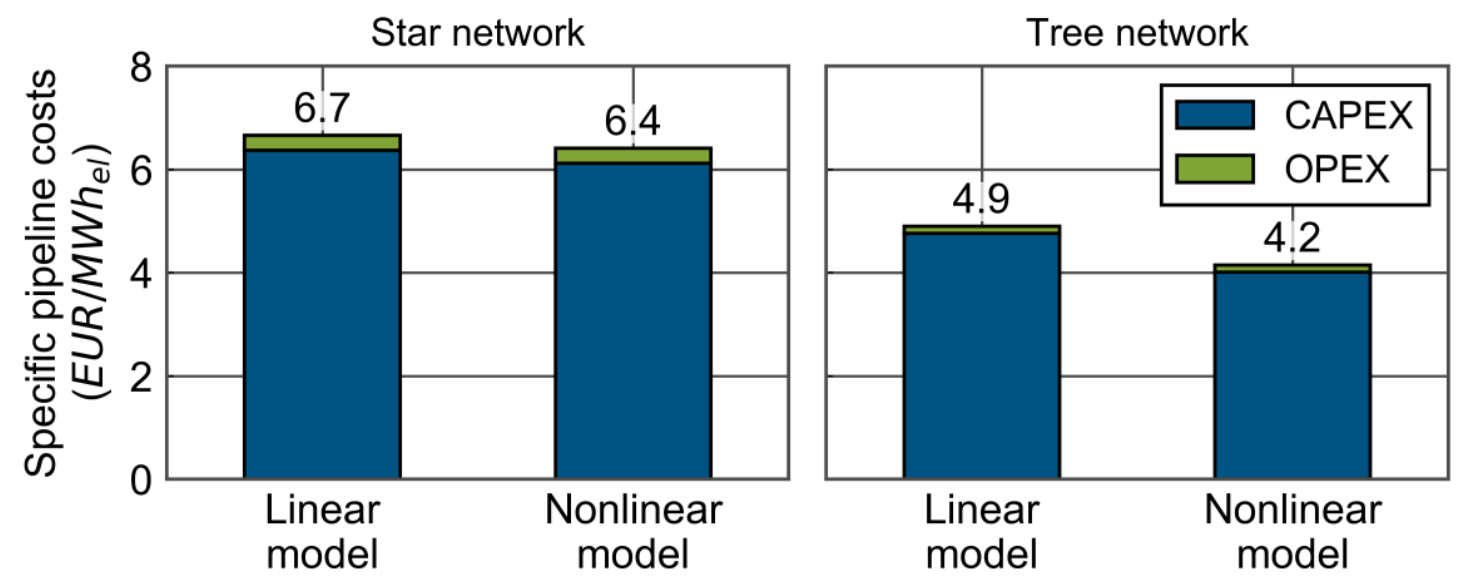

Figure 14. Comparison of specific pipeline costs for two different networks and two different flow modelling approaches. Total re-electrification costs from Welder et al. [9] for the CCGT scenario are 176 EUR/MWhel.

Indeed, this result highly depends on the scenario assumptions. The overall hydrogen demand is 57 TWh of hydrogen for yearly electrical reconversion, which amounts to roughly 2.6 million tons of hydrogen per year. Reuß et al. [7] compute an overall demand of 3.03 million tons of hydrogen for supplying German passenger cars with $75 \%$ of the share of fuel cell electric vehicles. This indicates that the overall hydrogen demand in this study is high and leads to low costs. Lowering the overall demand will lead to decreased pipeline diameters and therefore higher specific costs of hydrogen [5,7]. It should be noted though that with decreased diameters, the relative differences between the linear and nonlinear model will decline as well. To adequately analyze this issue, further considerations are necessary in future studies.

Finally, the resulting diameters obtained from the linear model are used as inputs in the nonlinear model and checked for their feasibility. To do so, the pipeline costs for the respective diameters from the linear result were set to zero. If the model opts to build a larger pipeline, the results obtained from the linear model are infeasible for the nonlinear constraints of pressure drop. In Figure 15, the results for the feasibility check of the star network and the tree network are given. In the star network, three pipes are not feasible and the pipeline diameter is increased. For the tree network, just one pipe is changed. However, none of both pipeline systems is entirely feasible. This represents an important benefit of the nonlinear model, as its results are closer to reality.

\section{Conclusion}

Future energy systems based on renewable energy technologies could heavily rely on hydrogen as a key element for large-scale storage, sector coupling and even electrical reconversion. Therefore, hydrogen pipelines are often used in energy system optimization models as a large-scale transport option. However, an optimized cost calculation of hydrogen flow through pipelines with respect to pressure drops is highly nonlinear and difficult to integrate into optimization models. Thus, it is often simplified in the literature. This contribution aims at evaluating the impact of this simplification on the feasibility and pipeline costs. To this end, a simplified topology from the literature is selected that is reproducible and analyzed. Based on this topology, two different network setups, namely a star network and tree network, are generated. With these two networks, two different flow modeling approaches were applied: a simplified fluid flow consideration with a fixed velocity and a cost calculation taking the pressure drop into account. 

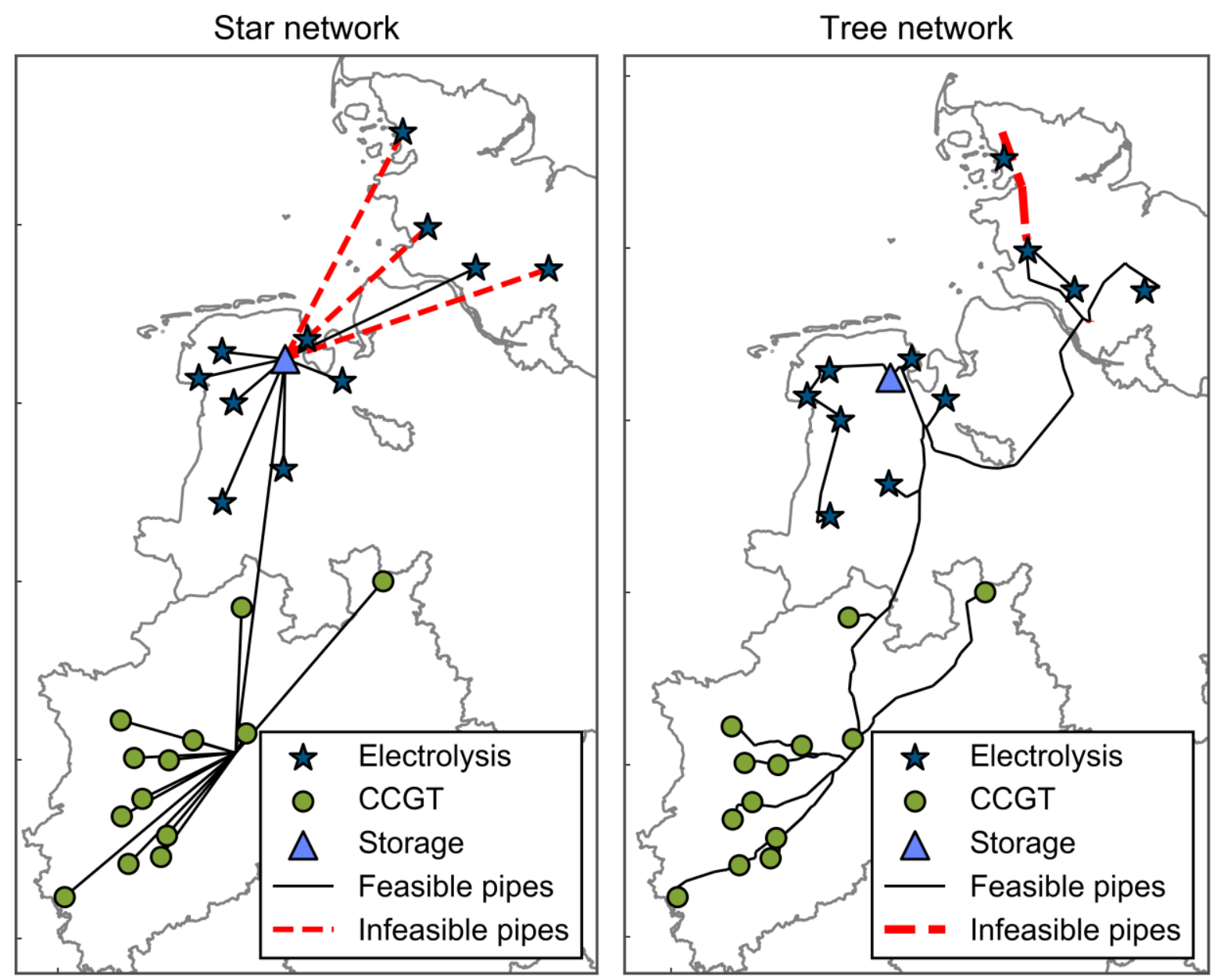

Figure 15. Feasibility check for the pipeline diameters from the linear model for the star network and the tree network with the nonlinear model.

The main result of this study is that a network with an increased number of nodes to fork pipes significantly lowers the total pipeline length, as well as the costs with the same system design approach. The consideration of a nonlinear pressure drop for the cost calculation with discrete diameters showed additional cost benefits compared to the linearized cost computation that utilizes a fixed gas velocity for the flow calculation. The differences between the assumed gas velocity and the resulting gas velocity chosen by the nonlinear model increases for larger diameters based on the obtained results. Consequently, pipelines with increasing hydrogen flows tend to be overestimated by linearizing the investment depending on the hydrogen flow. In summary, a drop of up to $37 \%$ in pipeline investment can be observed. However, the impact on the costs of electrical reconversion is almost not influenced, as the cost of renewable electricity and the respective efficiencies are significantly more expensive than the transport infrastructure. In such cases, the high computational effort for the in-depth representation of physical gas flow seems very high.

Based on these results, the utilization of nonlinear approaches for pressure drop consideration of pipeline flows, as well as increasing the number of network nodes, is relevant as it allows for the identification of the drawbacks of model simplification. However, the utilization of such approaches in energy system optimization models is always a trade-off between computational performance and the necessary degree of detail. Meanwhile, the integration of nonlinearities into energy system optimization models cannot be expected due to their high computational burden. Nevertheless, application as part of post-processing would strengthen the results and improve the credibility of future analyses. 


\section{Acknowledgements}

This work was supported by the Helmholtz Association under the Joint Initiative, "EnergySystem 2050 - A Contribution of the Research Field Energy". Moreover, this research has been performed as part of the Energie Campus Nürnberg and is supported by funding of the Bavarian State Government. Furthermore, the authors acknowledge the financial support by the Federal Ministry for Economic Affairs and Energy of Germany in the project METIS (project numbers 03ET4064A and 03ET4064C). The third, sixth, and seventh author also thank the DFG for their support within projects A05, B07, and B08 of the CRC TRR 154.

\section{References}

[1] UN. Adoption of the Paris Agreement. Framework Convention on Climate Change. Paris: United Nations; 2015.

[2] Roberts D. A global roadmap for climate change action: From COP17 in Durban to COP21 in Paris. S Afr J Sci. 2016;112:9-11.

[3] Robinius M, Otto A, Heuser P, Welder L, Syranidis K, Ryberg D, Grube T, Markewitz P, Peters R, Stolten D. Linking the Power and Transport Sectors-Part 1: The Principle of Sector Coupling. Energies. 2017;10:956.

[4] Bloess A, Schill W-P, Zerrahn A. Power-to-heat for renewable energy integration: A review of technologies, modeling approaches, and flexibility potentials. Applied Energy. 2018;212:1611-26.

[5] Yang C, Ogden J. Determining the lowest-cost hydrogen delivery mode. International Journal of Hydrogen Energy. 2007;32:268-86.

[6] Baufumé S, Grüger F, Grube T, Krieg D, Linssen J, Weber M, Hake J-F, Stolten D. GIS-based scenario calculations for a nationwide German hydrogen pipeline infrastructure. International Journal of Hydrogen Energy. 2013;38:3813-29.

[7] Reuß M, Grube T, Robinius M, Stolten D. A hydrogen supply chain with spatial resolution: Comparative analysis of infrastructure technologies in Germany. Applied Energy. 2019;247:438-53.

[8] Welder L, Ryberg DS, Kotzur L, Grube T, Robinius M, Stolten D. Spatio-temporal optimization of a future energy system for power-to-hydrogen applications in Germany. Energy. 2018.

[9] Welder L, Stenzel P, Ebersbach N, Markewitz P, Robinius M, Emonts B, Stolten D. Design and evaluation of hydrogen electricity reconversion pathways in national energy systems using spatially and temporally resolved energy system optimization. International Journal of Hydrogen Energy. 2018.

[10] Samsatli S, Staffell I, Samsatli NJ. Optimal design and operation of integrated wind-hydrogen-electricity networks for decarbonising the domestic transport sector in Great Britain. International Journal of Hydrogen Energy. 2016;41:447-75.

[11] Samsatli S, Samsatli NJ. The role of renewable hydrogen and inter-seasonal storage in decarbonising heat - Comprehensive optimisation of future renewable energy value chains. Applied Energy. 2019;233-234:854-93.

[12] Moreno-Benito M, Agnolucci P, Papageorgiou LG. Towards a sustainable hydrogen economy: Optimisation-based framework for hydrogen infrastructure development. Computers \& Chemical Engineering. 2017;102:110-27.

[13] Almansoori A, Betancourt-Torcat A. Design of optimization model for a hydrogen supply chain under emission constraints - A case study of Germany. Energy. 2016;111:414-29. 
[14] Ochoa Bique A, Zondervan E. An outlook towards hydrogen supply chain networks in 2050 - Design of novel fuel infrastructures in Germany. Chemical Engineering Research and Design. 2018;134:90-103.

[15] Weber AC, Papageorgiou LG. Design of hydrogen transmission pipeline networks with hydraulics. Chemical Engineering Research and Design. 2018;131:266-78.

[16] Groissböck M. Are open source energy system optimization tools mature enough for serious use? Renewable and Sustainable Energy Reviews. 2019;102:234-48.

[17] Robinius M, Schewe L, Schmidt M, Stolten D, Thürauf J, Welder L. Robust optimal discrete arc sizing for tree-shaped potential networks. Computational Optimization and Applications. 2019;73:1-29.

[18] Schmidt M, Steinbach MC, Willert BM. High detail stationary optimization models for gas networks: validation and results. Optimization and Engineering. 2016;17:43772.

[19] Schmidt M, Steinbach MC, Willert BM. High detail stationary optimization models for gas networks. Optimization and Engineering. 2015;16:131-64.

[20] Aßmann D, Liers F, Stingl M. Decomposable robust two-stage optimization: An application to gas network operations under uncertainty. Networks. 2019;74:40-61.

[21] Gorissen BL, Yanıkoğlu I, den Hertog D. A practical guide to robust optimization. Omega. 2015;53:124-37.

[22] André J, Auray S, Brac J, De Wolf D, Maisonnier G, Ould-Sidi M-M, Simonnet A. Design and dimensioning of hydrogen transmission pipeline networks. European Journal of Operational Research. 2013;229:239-51.

[23] Dijkstra E. A note on two problems in connexion with graphs. Numerische Mathematik. 1959;1:269-71.

[24] Weymouth TR. Problems in Natural Gas Engineering. Transactions of the ASME. $1912 ; 34$.

[25] Gross M, Pfetsch ME, Schewe L, Schmidt M, Skutella M. Algorithmic results for potential-based flows: Easy and hard cases. Networks. 2019;73:306-24.

[26] Fügenschuh A, Geißler B, Gollmer R, Morsi A, Pfetsch ME, Rövekamp J, Schmidt M, Spreckelsen K, Steinbach MC. Physical and technical fundamentals of gas networks. In: Koch T, Hiller B, Pfetsch ME, Schewe L, editors. Evaluating Gas Network Capacities: SIAM; 2015. p. 17-44.

[27] Lenz R, Schwarz R. Optimal Looping of Pipelines in Gas Networks. ZIB Report (16-67): Zuse Institut Berlin; 2016.

[28] Mischner J, Fasold H-G, Kadner K. gas2energy.net: Systemplanerische Grundlagender Gasversorgung: Deutscher Industrieverlag; 2011.

[29] Samsatli S, Samsatli NJ. A general spatio-temporal model of energy systems with a detailed account of transport and storage. Computers \& Chemical Engineering. 2015;80:155-76.

[30] Robinius M, Otto A, Syranidis K, Ryberg DS, Heuser P, Welder L, Grube T, Markewitz P, Tietze V, Stolten D. Linking the Power and Transport Sectors-Part 2: Modelling a Sector Coupling Scenario for Germany. Energies. 2017;10:957.

[31] Welder L, Linßen J, Robinius M, Stolten D. FINE - Framework for Integrated Energy System Assessment. v.0.0.1 ed. available at http://github.com/FZJ-IEK3VSA/FINE 2018. 